\title{
Gradient of the stellar magnetic field in measurements of hydrogen line cores
}

\author{
Dimitry O. Kudryavtsev and Iosif I. Romanyuk \\ Special Astrophysical Observatory RAS, Nizhny Arkhyz, 369167, Russia
}

\begin{abstract}
We report the observed systematic differences in longitudinal magnetic field values, obtained from measurements of metal lines and the core of the $\mathrm{H}_{\beta}$ line for a number of $\mathrm{Ap}$ stars, having strong global magnetic fields. In overwhelming majority of cases the magnetic field values, obtained from measurements of hydrogen lines cores, is smaller then the ones obtained from metal lines. We discuss some possible explanations of this effect, the most probable of which is the existence of the gradient of the magnetic field in stellar atmospheres.
\end{abstract}

Keywords. Stars: magnetic field - stars: chemically peculiar - stars: 53 Cam

\section{Observations and measurements}

The Zeeman spectra have been obtained in three different observational runs in 2006, 2008. We used the Main Stellar Spectrograph of the $6 \mathrm{~m}$ telescope equipped with the image slicer and the circular polarization analyzer (Chountonov (2004)). The spectral resolution $R=15000$. For our observations we used the spectral range $4760-5000 \AA$, which includes 50-100 metal lines and the line $\mathrm{H}_{\beta}$. Longitudinal magnetic field values were measured by the Zeeman shift between lines positions in right-hand and left-hand circularly polarized spectra, using standard Babcock's formula. Positions of spectral lines were determined by the fitting of profiles with a Gaussian.

\section{The effect}

In Fig. 1 we show a region of a spectrum of the star 53 Cam in right-hand and left-hand circular polarizations, and the parameter Stokes $\mathrm{V}$ for this region. One can see that the Stokes $\mathrm{V}$ values in metal lines is greater than the Stokes $\mathrm{V}$ in $\mathrm{H}_{\beta}$. Particularly this effect is caused by the fact that metall lines have generally higher Lande factors $(z=1.23$ on average), and therefore are more sensitive to the magnetic field, while $\mathrm{H}_{\beta}$ has $z=1.0$. But in Zeeman measurements we take this effect into account, and nevertheless the measured longitudinal magnetic field $B_{e}$ shows sufficiently different values for metal lines and for the core of $\mathrm{H}_{\beta}$.

We have about 50 measurements for 30 stars, which unfortunately cannot be presented here due to the page limits. The core of the $\mathrm{H}_{\beta}$ line shows systematically lower values of $B_{e}$ for almost all the cases when the longitudinal magnetic field differs from the zero.

\section{Possible explanations}

Partial Zeeman splitting. Metal lines generally have more complex picture of Zeeman splitting than the $\mathrm{H}_{\beta}$. For stars with strong fields this may cause the asymmetry or the partial splitting of spectral lines. In those cases our procedure of the line center determination by fitting a Gaussian may cause a fault. But with the moderate spectral 


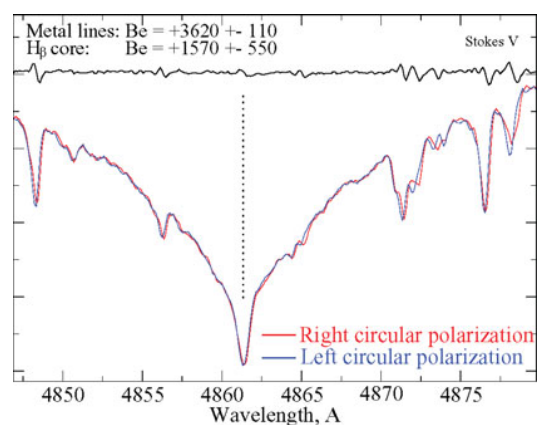

Figure 1. A spectrum of right, left circular polarizations and Stokes V for the star 53 Cam.

resolution we used, this could be true only for stars with a very strong surface magnetic fields. But for most our stars the metal lines are symmetrical for the resolution $R=15000$ and no partial Zeeman splitting is observed.

Spots. Ap stars are well known as the objects with the inhomogeneous distribution of chemical elements over the surface. This could influence the magnetic field measurements. If the measured lines belong to an element concentrated near magnetic poles than the measured $B_{e}$ will be higher than the $B_{e}$, obtained from hydrogen lines. Metal lines, we used for measurements, are mostly the lines of Fe and Cr. Though these elements could have inhomogeneous distribution, they are present enough over all the surface of a star and actually for most cases the spectral variability is not so great during rotational period. As an evidence of low influence of spots to our measurements we refer to the measurements of $53 \mathrm{Cam}$ and $\gamma \mathrm{Equ}$, where our measurements of metal lines corresponds well with the measurements of other authors, obtained by measuring of polarization in wings of hydrogen lines.

Gradient of the magnetic field. Another way to explain why the measured longitudinal magnetic fields differ, is to suggest that we observe the gradient of the magnetic field in stellar atmosphere. The forming of metall lines and the cores of hydrogen lines takes place in different layers of the stellar atmosphere. So if there is a difference in magnetic field strengths between these layers we must observe different values of the longitudinal magnetic field. Accepting this explanation, we should note that the observed gradient of the magnetic field is not represent usual magnetic field decreasing with the moving out of the center of a dipole as the thickness of the stellar atmosphere is too small for this effect to be observed. Thus, probably we observe a strong deviation from the standard dipole model of the magnetic fields of Ap stars within the rather small radial scale.

\section{Resume}

Although the partial Zeeman splitting and inhomogeneous chemical abundances could explain the difference between measured magnetic field values in some special cases, there is good reason to believe that they are not sufficient to describe all our measurements. We suppose that there is the influence of the radial gradient of the magnetic field in the stellar atmosphere having place in our measurements of cores of hydrogen lines, at least partially.

\section{References}

Chountonov, G. A. 2004, in: Yu. V. Glagolevskij, D. O. Kudryavtsev, \& I. I. Romanyuk (eds.), Magnetic stars, Proc. International Conf. (Nizhny Arkhyz: SAO Press), p. 289 